\title{
Fast Discrete Chirp Fourier Transforms For Radar Signal Detection Systems Using Cluster Computer Implementations
}

\author{
Cesar A Aceros-Moreno, Domingo Rodriguez \\ Automated Information Processing Laboratory \\ Electrical and Computer Engineering \\ University of Puerto Rico at Mayaguez \\ Mayaguez, PR, 00681-9042 \\ e-mail: \{cesar.aceros,domingo\}@ece.uprm.edu
}

\begin{abstract}
A useful processing tool for time-frequency signals known as the Discrete Chirp Fourier Transform (DCFT) and its implementation using cluster computers for application to RADAR signal analysis. A time-frequency signal is defined as a signal whose spectral distribution changes with time. Chirp signals, as time-frequency signals which are linearly frequency modulated, are widely used in many detection, estimation, and imaging RADAR applications. DCFT implementations for RADAR systems provide important information about the nature of radar signals and allow to determine certain spectral characteristics used in signal detection and estimation processes.
\end{abstract}

Index Terms-Chirp Transform, MPI Cluster, Fast Transform

\section{INTRODUCTION}

$\mathbf{T}$ HIS his work concentrates on the analysis, design, and implementation of efficient algorithms for the computation of the DCFT. A DCFT can be thought of as the discrete Fourier transform(DFT) of a signal which has being multiplied by a chirp signal and, hence, being shifted in the spectral domain. The algorithms involve mathematical tools, such as Kronecker products algebra and finite abelian group theory in order to express DCFT in terms of factored composition of sparse matrices. A DCFT takes a one-dimensional signal in the object domain and returns a two-dimensional signal in the chirp transform or spectral domain. Two cases have been used for DCFT algorithm development. A first case is (Fast Fourier Transform) FFT-like and expresses the DCFT when the lenght is a composite number. A second case we treat when the lenght of the signal is a prime number.

\section{A. Chirp Fourier Transform}

The 1-D DCFT [1] is defined as:

$$
X_{c}[k, l]=\frac{1}{\sqrt{N}} \sum_{n=0}^{N-1} x[n] W_{N}^{l n^{2}+k n} \quad, 0 \leq k, l \leq N-1
$$

From the definition of the DCFT two parameters ( $l$ and $k$ ) are into equation 1 . The $k$ parameter is the same parameter of the Discrete Time Fourier Transform(DTFT). The $l$ parameter generates an $l n^{2}$ product resulting in a new independent variable of the transform function. This expansion means more products and finally a higher computational effort.

\section{B. Kronecker Product Definition}

Let $\mathrm{A}$ be an $n \times p$ matrix and $\mathrm{B}$ an $m \times q$ matrix.

$$
A=\left[\begin{array}{cccc}
a_{0,0} & a_{0,1} & \cdots & a_{0, p-1} \\
a_{1,0} & a_{1,1} & \cdots & a_{1, p-1} \\
\vdots & \vdots & \ddots & \vdots \\
a_{n-1,0} & a_{n-1,1} & & a_{n-1, p-1}
\end{array}\right]
$$

then,

$$
A \otimes B=\left[\begin{array}{cccc}
a_{0,0} \cdot B & a_{0,1} \cdot B & \cdots & a_{0, p-1} \cdot B \\
a_{1,0} \cdot B & a_{1,1} \cdot B & \cdots & a_{1, p-1} \cdot B \\
\vdots & \vdots & \ddots & \vdots \\
a_{n-1,0} \cdot B & a_{n-1,1} \cdot B & \cdots & a_{n-1, p-1} \cdot B
\end{array}\right]_{m \cdot n \times p \cdot q}
$$

The $m \cdot n \times p \cdot q$ matrix is called the Kronecker product of A and B. It is also called the direct product or the tensor product.

An important property of the Tensor product is that for conforming matrices, $(A \otimes B)(C \otimes D)=A C \otimes B D$.

\section{FFT Representation in Terms of Kronecker Product}

By definition the Discrete Time Fourier Transform (DTFT) is defined as:

$$
X[k]=\underbrace{\frac{1}{\sqrt{N}} \sum_{n=0}^{N-1} W_{N}^{k n}}_{F(N)} x[n], 0 \leq k \leq N-1
$$

And the DTFT of the sequence $x[n]$, is defined for the following matrix-vector product.

$$
X=F(N) \cdot x
$$


Using group theory and Kronecker product the $F(N)$ matrix is possible be expressed by the following expression [2]:

If $\mathrm{N}=\mathrm{RS}$, then:

$$
F(N)=\left(F(R) \otimes I_{S}\right) T_{R, S}\left(I_{R} \otimes F(S)\right) P_{N, R}
$$

This is a simplification because we are representing a high order $F(N)$ in terms of the lower order matrices $F(R)$ and $F(S)$.

D. Definition of the Multiplicative Group Permutation Matrix

Let $v$ denote the following function.

$$
\begin{aligned}
v: \mathbb{Z}_{7}^{\times} & \longrightarrow G_{7,5} \\
k & \longmapsto v(k) \equiv\left\langle 5^{k}\right\rangle_{7}
\end{aligned}
$$

Thus, following the natural order of $\mathbb{Z}_{7}^{\times}$, we obtain

$$
\begin{aligned}
& G_{7,5}=\left\{\left\langle 5^{1}\right\rangle_{7},\left\langle 5^{2}\right\rangle_{7},\left\langle 5^{3}\right\rangle_{7},\left\langle 5^{4}\right\rangle_{7},\left\langle 5^{5}\right\rangle_{7},\left\langle 5^{6}\right\rangle_{7}\right\} \\
& G_{7,5}=\{546231\}
\end{aligned}
$$

Using the prescribed order of $G_{7,5}$ to index the rows and columns of the table, except for column zero or row zero. The number 5 is known as a generator of $(\mathbb{Z} / 7 \mathbb{Z})^{x}$ This is the same that apply the following permutation matrix [3]:

$$
P_{G_{7,5}}=\left[\begin{array}{ccccccc}
1 & 0 & 0 & 0 & 0 & 0 & 0 \\
0 & 0 & 0 & 0 & 0 & 1 & 0 \\
0 & 0 & 0 & 0 & 1 & 0 & 0 \\
0 & 0 & 0 & 0 & 0 & 0 & 1 \\
0 & 0 & 1 & 0 & 0 & 0 & 0 \\
0 & 0 & 0 & 1 & 0 & 0 & 0 \\
0 & 1 & 0 & 0 & 0 & 0 & 0
\end{array}\right]
$$

\section{DCFT REPRESENTATION AS A MATRIX-VECTOR PRODUCT}

The equation 1, could be rewritten as a matrix-vector product for the specific case $N=8$.

$$
X_{c}[k, l]=[\boldsymbol{K}(\boldsymbol{8})] \cdot\left[\begin{array}{c}
x[0] \\
x[1] W_{N}^{l} \\
x[2] W_{N}^{4 l} \\
x[3] W_{N}^{9 l} \\
x[4] W_{N}^{16 l} \\
x[5] W_{N}^{25 l} \\
x[6] W_{N}^{36 l} \\
x[7] W_{N}^{49 l}
\end{array}\right]
$$

And in a general form for length $\mathrm{N}$.

$$
X_{c}[k, l]=F(N) \cdot\left[x[n] \odot_{N} W_{N}^{l n^{2}}\right]
$$

where $\odot$ denotes Haddamard product.

\section{A. Volumetric Representation of the DCFT}

In the same way as the DTFT can be seen as a matrixvector product, the DCFT can be seen as a product of a Cubic matrix-vector product. See figure 1.

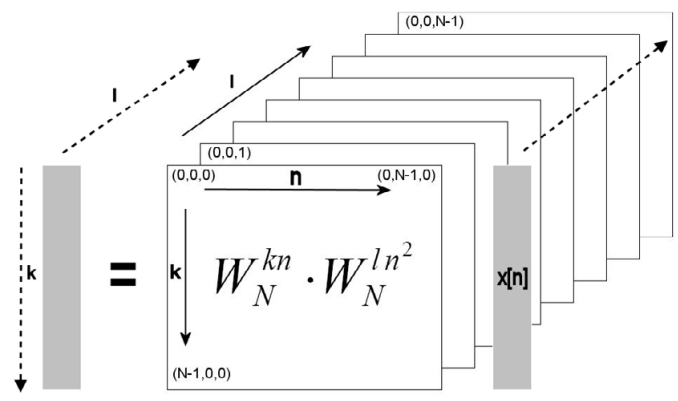

Fig. 1. Index variation

Expanding the $N$ x $N$ x Chirp cube we have a layer representation in terms of the $l$ index. The $C_{4}[l]$ is defined as follow:

$$
\begin{gathered}
C_{4}[l]=\left[\left[\begin{array}{cccc}
1 & 1 & 1 & 1 \\
1 & W_{4} & W_{4}^{2} & W_{4}^{3} \\
1 & W_{4}^{2} & 1 & W_{4}^{2} \\
1 & W_{4}^{3} & W_{4}^{2} & W_{4}
\end{array}\right],\left[\begin{array}{cccc}
1 & W_{4} & 1 & W_{4} \\
1 & W_{4}^{2} & W_{4}^{2} & 1 \\
1 & W_{4}^{3} & 1 & W_{4}^{3} \\
1 & 1 & W_{4}^{2} & W_{4}^{2}
\end{array}\right],\right. \\
\left.\left[\begin{array}{cccc}
1 & W_{4}^{2} & 1 & W_{4}^{2} \\
1 & W_{4}^{3} & W_{4}^{2} & W_{4} \\
1 & 1 & 1 & 1 \\
1 & W_{4} & W_{4}^{2} & W_{4}^{3}
\end{array}\right],\left[\begin{array}{cccc}
1 & W_{4}^{3} & 1 & W_{4}^{3} \\
1 & 1 & W_{4}^{2} & W_{4}^{2} \\
1 & W_{4} & 1 & W_{4} \\
1 & W_{4}^{2} & W_{4}^{2} & 1
\end{array}\right]\right]
\end{gathered}
$$

\section{FFT-LIKE APPROACH TO THE DCFT}

Is important note that:

$$
C_{4}[1]=F(4)=\left[\begin{array}{cccc}
1 & 1 & 1 & 1 \\
1 & W_{4} & W_{4}^{2} & W_{4}^{3} \\
1 & W_{4}^{2} & 1 & W_{4}^{2} \\
1 & W_{4}^{3} & W_{4}^{2} & W_{4}
\end{array}\right]
$$

Now considering the Permutation Matrix $P_{4,2}$

$$
P_{4,2}=\left[\begin{array}{cccc}
1 & 0 & 0 & 0 \\
0 & 0 & 1 & 0 \\
0 & 1 & 0 & 0 \\
0 & 0 & 0 & 1
\end{array}\right]
$$

(9) We can apply this transformation to the rows and columns of the whole Chirp Matrix (equation 11), we obtain the following result:

$$
C_{4 P}[l]=P_{4,2}^{-1} \cdot C_{4}[l] \cdot P_{4,2}
$$


Theorem 1: If $\mathrm{N}$ is power of 2.

$$
\begin{aligned}
& C_{4 P}[l]=\left[\begin{array}{cccc}
1 & 1 & 1 & 1 \\
1 & 1 & W_{4}^{2} & W_{4}^{2} \\
1 & W_{4}^{2} & W_{4} & W_{4}^{3} \\
1 & W_{4}^{2} & W_{4}^{3} & W_{4}
\end{array}\right],\left[\begin{array}{cccc}
1 & 1 & W_{4} & W_{4} \\
1 & 1 & W_{4}^{3} & W_{4}^{3} \\
1 & W_{4}^{2} & W_{4}^{2} & 1 \\
1 & W_{4}^{2} & 1 & W_{4}^{2}
\end{array}\right] \text { Where, } C_{N}[l]=\left(F_{\frac{N}{2}} \otimes I_{2}\right) T_{\frac{N}{2}, 2}\left(\Gamma_{N, 2}^{\prime}[l] \otimes F_{2}\right) \cdot P_{N, 2}^{-1} \\
& \left.\left[\begin{array}{cccc}
1 & 1 & W_{4}^{2} & W_{4}^{2} \\
1 & 1 & 1 & 1 \\
1 & W_{4}^{2} & W_{4}^{3} & W_{4} \\
1 & W_{4}^{2} & W_{4} & W_{4}^{3}
\end{array}\right],\left[\begin{array}{cccc}
1 & 1 & W_{4}^{3} & W_{4}^{3} \\
1 & 1 & W_{4} & W_{4} \\
1 & W_{4}^{2} & 1 & W_{4}^{2} \\
1 & W_{4}^{2} & W_{4}^{2} & 1
\end{array}\right]\right] \quad(15) \\
& \text { And now multiplying each one of the matrices of the }
\end{aligned}
$$
equation 15 for the following Transformation Matrices:

$$
\begin{aligned}
& \Gamma_{4,2}[l]=\left[\begin{array}{cccc}
1 & 0 & 0 & 0 \\
0 & 1 & 0 & 0 \\
0 & 0 & W_{4}^{0} & 0 \\
0 & 0 & 0 & W_{4}^{0}
\end{array}\right],\left[\begin{array}{cccc}
1 & 0 & 0 & 0 \\
0 & 1 & 0 & 0 \\
0 & 0 & W_{4}^{1} & 0 \\
0 & 0 & 0 & W_{4}^{1}
\end{array}\right], \\
& \left.\left[\begin{array}{cccc}
1 & 0 & 0 & 0 \\
0 & 1 & 0 & 0 \\
0 & 0 & W_{4}^{2} & 0 \\
0 & 0 & 0 & W_{4}^{2}
\end{array}\right],\left[\begin{array}{cccc}
1 & 0 & 0 & 0 \\
0 & 1 & 0 & 0 \\
0 & 0 & W_{4}^{3} & 0 \\
0 & 0 & 0 & W_{4}^{3}
\end{array}\right]\right] \\
& \Gamma_{4,2}[l]=\left[\left[\begin{array}{cc}
1 & 0 \\
0 & W_{4}^{0}
\end{array}\right] \otimes I_{2},\left[\begin{array}{cc}
1 & 0 \\
0 & W_{4}^{1}
\end{array}\right] \otimes I_{2},\right. \\
& \left.\left[\begin{array}{cc}
1 & 0 \\
0 & W_{4}^{2}
\end{array}\right] \otimes I_{2},\left[\begin{array}{cc}
1 & 0 \\
0 & W_{4}^{3}
\end{array}\right] \otimes I_{2}\right] \\
& \Gamma_{4,2}[l]=\left[\begin{array}{cc}
1 & 0 \\
0 & W_{4}^{l}
\end{array}\right] \otimes I_{2} \\
& =\Gamma_{4,2}^{\prime}[l] \otimes I_{2}
\end{aligned}
$$

We must take notice of equation 12 that:

$$
\begin{aligned}
C_{4 P}[l] & =C_{4 P}[1] \cdot \Gamma_{4,2}[l] \\
C_{4 P}[l] & =P_{4,2}^{-1} \cdot C_{4}[1] \cdot P_{4,2} \cdot \Gamma_{4,2}[l] \\
P_{4,2}^{-1} \cdot C_{4}[l] \cdot P_{4,2} & =P_{4,2}^{-1} \cdot C_{4}[1] \cdot P_{4,2} \cdot \Gamma_{4,2}[l] \\
C_{4}[l] & =\underbrace{P_{4,2} \cdot P_{4,2}^{-1}}_{I_{4}} \cdot C_{4}[1] \cdot P_{4,2} \cdot \Gamma_{4,2}[l] \cdot P_{4,2}^{-1} \\
C_{4}[l] & =F_{4} \cdot P_{4,2} \cdot \Gamma_{4,2}[l] \cdot P_{4,2}^{-1}
\end{aligned}
$$

But using equation 6 ,

$$
F_{4}=\left(F_{2} \otimes I_{2}\right) \cdot T_{2,2} \cdot\left(I_{2} \otimes F_{2}\right) \cdot P_{4,2}
$$

And replacing in 20

$$
\begin{aligned}
C_{4}[l] & =\left(F_{2} \otimes I_{2}\right) T_{2,2}\left(I_{2} \otimes F_{2}\right) \underbrace{P_{4,2} \cdot P_{4,2}}_{I_{4}} \cdot \Gamma_{4,2}[l] P_{4,2}^{-1} \\
& =\left(F_{2} \otimes I_{2}\right) T_{2,2}\left(I_{2} \otimes F_{2}\right) \cdot \Gamma_{4,2}[l] P_{4,2}^{-1} \\
& =\left(F_{2} \otimes I_{2}\right) T_{2,2}\left(I_{2} \otimes F_{2}\right) \cdot\left(\Gamma_{4,2}^{\prime}[l] \otimes I_{2}\right) \cdot P_{4,2}^{-1}
\end{aligned}
$$

By direct computation, a general form for the $C_{N}[l]$ can be obtained using the following theorem:

\section{DCFT For Prime Number of S AMPLes}

For N=5. From definition I-D, we found that 3 is a generator of $G_{5,3}$.

$$
\begin{aligned}
G_{5,3} & =\left\{\left\langle 3^{1}\right\rangle_{5},\left\langle 3^{2}\right\rangle_{5},\left\langle 3^{3}\right\rangle_{5},\left\langle 3^{4}\right\rangle_{5}\right\} \\
G_{5,3} & =\{3421\}
\end{aligned}
$$

This define the following permutation matrix:

$$
P_{G_{5,3}}=\left[\begin{array}{ccccc}
1 & 0 & 0 & 0 & 0 \\
0 & 0 & 0 & 1 & 0 \\
0 & 0 & 0 & 0 & 1 \\
0 & 0 & 1 & 0 & 0 \\
0 & 1 & 0 & 0 & 0
\end{array}\right]
$$

The $C_{5}[1]$ is defined as:

$$
C_{5}[1]=\left[\begin{array}{ccccc}
1 & 1 & 1 & 1 & 1 \\
1 & W_{5} & W_{5}^{2} & W_{5}^{3} & W_{5}^{4} \\
1 & W_{5}^{2} & W_{5}^{4} & W_{5} & W_{5}^{3} \\
1 & W_{5}^{3} & W_{5} & W_{5}^{4} & W_{5}^{2} \\
1 & W_{5}^{4} & W_{5}^{3} & W_{5}^{2} & W_{5}
\end{array}\right]
$$

Applying the permutation matrix $P_{G_{5,3}}$ to all the rows and columns of the $C_{5}[l]$ matrix we obtain the $C_{5 r}[l]$ matrix.

$$
C_{5 r}[l]=P_{G_{5,3}} \cdot C_{5}[l] \cdot P_{G_{5,3}}^{-1}
$$

for $l=0$ we got,

$$
C_{5 r}[1]=\left[\begin{array}{ccccc}
1 & 1 & 1 & 1 & 1 \\
1 & W_{5} & W_{5}^{2} & W_{5}^{4} & W_{5}^{3} \\
1 & W_{5}^{2} & W_{5}^{4} & W_{5}^{3} & W_{5} \\
1 & W_{5}^{4} & W_{5}^{3} & W_{5} & W_{5}^{2} \\
1 & W_{5}^{3} & W_{5} & W_{5}^{2} & W_{5}^{4}
\end{array}\right]
$$

The matrix above is interesting because if we eliminate the first row and column, leave a Hankel matrix.

To the other layers can be expressed in terms of the $l=0$ , with the use of the transformation matrix $H_{5}$ defined by,

$$
H_{5}[l]=\left[\begin{array}{ccccc}
1 & 0 & 0 & 0 & 0 \\
0 & W_{5}^{-4 l} & 0 & 0 & 0 \\
0 & 0 & W_{5}^{-l} & 0 & 0 \\
0 & 0 & 0 & W_{5}^{-4 l} & 0 \\
0 & 0 & 0 & 0 & W_{5}^{-l}
\end{array}\right]
$$




$$
\begin{aligned}
& C_{5 r}[0]=C_{5 r}[l] \cdot H_{5}[l] \\
& C_{5 r}[l]=C_{5 r}[0] \cdot\left(H_{5}[l]\right)^{-1}
\end{aligned}
$$

Substituting in the 26 that,

$$
C_{5}[l]=P_{G_{5,3}}^{-1} \cdot C_{5 r}[0] \cdot\left(H_{5}[l]\right)^{-1} \cdot P_{G_{5,3}}
$$

Now the matrix vector product for the data gives,

$$
C_{5}[l] \cdot x[n]=\left\{P_{G_{5,3}}^{-1} \cdot C_{5 r}[0] \cdot\left(H_{5}[l]\right)^{-1} \cdot P_{G_{5,3}}\right\} \cdot x[n]
$$

A lot of algorithms for the efficient computation of the matrix vector product of a Hankel matrix and vector have been developed [4].

By direct computation, a general form for the $C_{N}[l]$ can be obtained using the following theorem:

Theorem 2: If $\mathrm{N}$ is a prime number and $\mathrm{k}$ an integer generator of $G_{N, k}=\left\{\left\langle k^{1}\right\rangle_{N},\left\langle k^{2}\right\rangle_{N},\left\langle k^{3}\right\rangle_{N}, \ldots .,\left\langle k^{N-1}\right\rangle_{N}\right\}$, then

$$
C_{N}[l]=P_{G_{N, k}}^{-1} \cdot C_{N r}[1] \cdot H_{N}[l] \cdot P_{G_{N, k}}
$$

Proof: We prove the above theorem by direct computation in the following manner.

The permutation operation is define as follows:

$$
\sigma_{G_{N, k}}=\left[\begin{array}{cccccc}
0 & 1 & 2 & 3 & \cdots & N-1 \\
0 & \left\langle k^{1}\right\rangle_{N} & \left\langle k^{2}\right\rangle_{N} & \left\langle k^{1}\right\rangle_{N} & \cdots & \left\langle k^{N-1}\right\rangle_{N}
\end{array}\right]
$$

And the transformation matrix $H_{N}[l]$ is:

$$
H_{N}[l]=P_{G_{N, k}} \cdot\left[\begin{array}{cccc}
1 & 0 & \cdots & 0 \\
0 & W_{N}^{-l} & \cdots & 0 \\
0 & \vdots & \ddots & \vdots \\
0 & 0 & \cdots & W_{N}^{-(N-1)^{2} l}
\end{array}\right] \cdot P_{G_{N, k}}^{-1}
$$

\section{MPI IMPLEMENTATION OF THE DCFT}

For the implementation of the DCFT in MPI Cluster we have used the dataflow of the figure 2 .

The implementation runs on IBM Linux Cluster with the following specifications:

- Computing Nodes: 64 IBM xSeries Servers, dual processors

- Processors: 128 Intel Pentium III $1.26 \mathrm{GHz}$

- Memory: 53 GygaBytes

- Storage: 1 TeraByte

- Operating System: Linux RedHat 7.1

- Network: Ethernet Gigabit network and Cisco Switches

Several implementations in Matlab using PC was made and the best results are compare with the Cluster implementation of the DCFT.

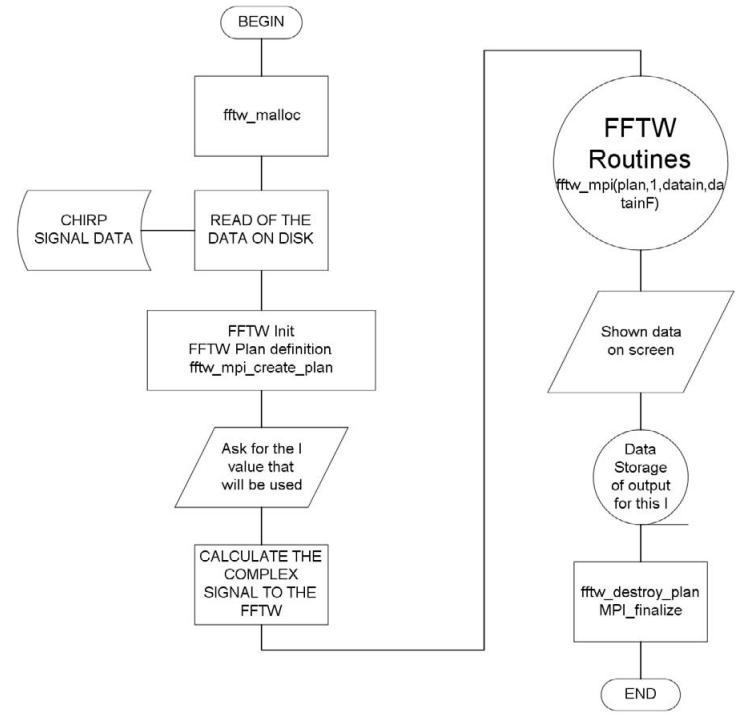

Fig. 2. Flow of MPI implementation

TABLE I

BENCHMARK TIME COMPARISON FOR CLUSTER IMPLEMENTATION

\begin{tabular}{|c|c|c|}
\hline Samples & $\begin{array}{c}\text { Sequential } \\
\text { Implementation } \\
\text { (seconds) }\end{array}$ & $\begin{array}{c}\text { Parallel } \\
\text { Implementation } \\
\text { (seconds) }\end{array}$ \\
\hline 64 & .06 & 0 \\
128 & .23 & 0.05 \\
256 & .90 & 0.21 \\
512 & 3.64 & 0.97 \\
1024 & 14.92 & 4.19 \\
2048 & 62.11 & 17.72 \\
4096 & Out of mem & 74.06 \\
8192 & Out of mem & 310.20 \\
\hline
\end{tabular}

\section{CONCLuSion}

The work that has been made in the development of DCFT algorithms is an useful issue. The case for prime numbers is special and is very interesting because the detection of the Chirp signal parameters is not an easy problem.

The formulation that have been made in this paper could be considered for implementation in other computational structures different to PC or cluster, for example Embedded systems or Field Programmable Gate Array (FPGA).

\section{REFERENCES}

[1] X.-G. Xia, "Discrete chirp-fourier transform and its application to chirp rate estimation.” IEEE Trans. Signal Processing, vol. 48, pp. 3122-3133, Nov 2000.

[2] D. Rodriguez, "Tensor product algebra as a tool for VLSI implementation of the discrete fourier transform," Proc. ICASSP, Toronto, ON, Canada, pp. 1025-1028, 1991.

[3] L. A. R. Tolimieri, "Ring structure and the fourier transform," The Mathemathical Intelligencer, vol. 7, no. 3, pp. 49-54, 1985.

[4] Z. T. R. D. N. A. Gumerov, "Fast algorithms to compute matrix-vector products for pascal matrices," UM Computer Science Department; CS-TR-4563 UMIACS; UMIACS-TR-2004-08, vol. https://drum.umd.edu/dspace/handle/1903/7, 25-Mar-2004. 